TITLE:

\title{
Experimental and numerical verification of microplasma assembly for novel electromagnetic media
}

\section{$\operatorname{AUTHOR}(\mathrm{S}):$}

Sakai, Osamu; Naito, Teruki; Tachibana, Kunihide

\section{CITATION:}

Sakai, Osamu ... [et al]. Experimental and numerical verification of microplasma assembly for novel electromagnetic media. PHYSICS OF PLASMAS 2010, 17(5): 057102.

\section{ISSUE DATE:}

2010-05

URL:

http://hdl.handle.net/2433/147221

\section{RIGHT:}

Copyright 2010 American Institute of Physics. This article may be downloaded for personal use only. Any other use requires prior permission of the author and the American Institute of Physics. The following article appeared in PHYSICS OF PLASMAS17, 057102 (2010) and may be found at 


\title{
Experimental and numerical verification of microplasma assembly for novel electromagnetic media ${ }^{a}$
}

\author{
Osamu Sakai, ${ }^{\text {b) }}$ Teruki Naito, and Kunihide Tachibana \\ Department of Electronic Science and Engineering, Kyoto University, Kyoto-daigaku Katsura, Nishikyo-ku, \\ Kyoto 615-8510, Japan
}

(Received 20 November 2009; accepted 18 January 2010; published online 26 March 2010)

\begin{abstract}
Microplasmas have a number of potential roles to control propagating electromagnetic waves. This report focuses on novel physics of periodic microplasma assembly for electromagnetic media, which is verified by experimental results and analyzed by numerical methods. Using an assembly composed of microplasmas, novel functions are expected due to its complex dielectric function arising from dielectric and lossy properties. The dielectric property creates photonic band gaps, and the lossy property drastically changes transmittance around the photonic band gaps, leading to an attenuation gap. As a result, a "complex" dispersion relation or a band diagram in the three-dimensional space of real and imaginary wavenumbers and wave frequency will open new possibilities to control electromagnetic waves by complex-variable filters composed of microplasma assemblies. () 2010 American Institute of Physics. [doi:10.1063/1.3314334]
\end{abstract}

\section{INTRODUCTION}

Microplasmas whose sizes are smaller than a few millimeters can give rise to functionality for chemical microreactors, conversion fields for biomaterials, and interactive media for photons. ${ }^{1,2}$ In particular, as far as interaction with photons or electromagnetic waves is concerned, in addition to microplasma generation and photon emission in an intensified electric field of waves, microplasmas can also play a number of potential roles of controllers for propagating waves. As a related scientific concern, dispersion relation of electromagnetic waves has been well understood in both magnetized and nonmagnetized plasmas, where they are assumed to be homogeneous, inhomogeneous within the regime of the Wentzel-Kramers-Brillouin approximation, or have specific parameter profiles in a cross section, such as that in a tokamak device. ${ }^{3-5}$ Recently, plasmas, which have abrupt spatial and temporal changes or are arranged in periodic structure, have attentions since they can serve as novel devices such as a frequency upshift converter, ${ }^{6}$ antenna, ${ }^{7}$ or a photonic crystal; ${ }^{8,9}$ we have already reported experimental verification of plasma photonic crystals composed of a microplasma array. $^{9-12}$

Theoretical approaches to reveal wave propagation in a periodical plasma assembly have been achieved so far in various methods. The Kronig and Penny model was useful for derivation of a band diagram in a one-dimensional (1D) plasma array. ${ }^{6,13}$ To simulate two-dimensional (2D) spatial periodicity, the plane-wave expansion method was modified for plasma array ${ }^{10}$ as well as for metallic structure ${ }^{14}$ to derive dispersion relation or a photonic band diagram, and this modified method can deal with collision effects as a loss term. Another method to calculate band diagrams of 2D structure is the direct complex-field analysis, ${ }^{10}$ and this

\footnotetext{
${ }^{\text {a) }}$ Paper GI2 1, Bull. Am. Phys. Soc. 54, 92 (2009).

${ }^{b)}$ Invited speaker.
}

method also allows us to analyze effects of a finite-size array. ${ }^{11}$

On the part of the experimental verification, we developed several types to realize $1 \mathrm{D}$ and $2 \mathrm{D}$ periodic plasma assemblies, using discharge extension of a microplasma array, ${ }^{9,15,16}$ a discharge assembly in multicapillary electrodes, ${ }^{11,17}$ and discharges in cold cathode fluorescent lamps (CCFLs). ${ }^{18-20}$ Self-organized discharge patterns in parallel-plate dielectric barrier discharges ${ }^{21-23}$ are simple and also promising regimes to realize a homogeneous $2 \mathrm{D}$ plasma assembly.

One missing point in the previous reports so far was permittivity working as a complex variable. When we use a collisional plasma instead of a collisionless plasma, the permittivity becomes a complex value, and we expect a new complex-variable filter made of plasma assemblies; collisions in plasmas give rise to imaginary part of the permittivity, and so we can expect independent control of wave amplitude and phase shift by changing electron density and gas pressure which determines electron elastic collision frequency. In addition, since we can control the frequency of the band gaps by varying spatial periodicity of the turn-on plasmas, we will obtain an elaborate tool for control of wave propagation.

This report focuses on novel physics of plasma assembly in a periodic configuration for electromagnetic media. When we make an assembly composed of microplasmas generated in a discharge scheme in which the working gas pressure is around the so-called Paschen-minimum condition, novel functions are expected due to its complex dielectric function arising from dielectric and lossy properties; the dielectric property creates photonic band gaps and the lossy property drastically changes transmittance around the photonic band gaps. In such cases, a "complex" dispersion relation or a complex band diagram describes electromagnetic-wave propagation in the three-dimensional (3D) space of real and imaginary wavenumbers and wave frequency, which will 


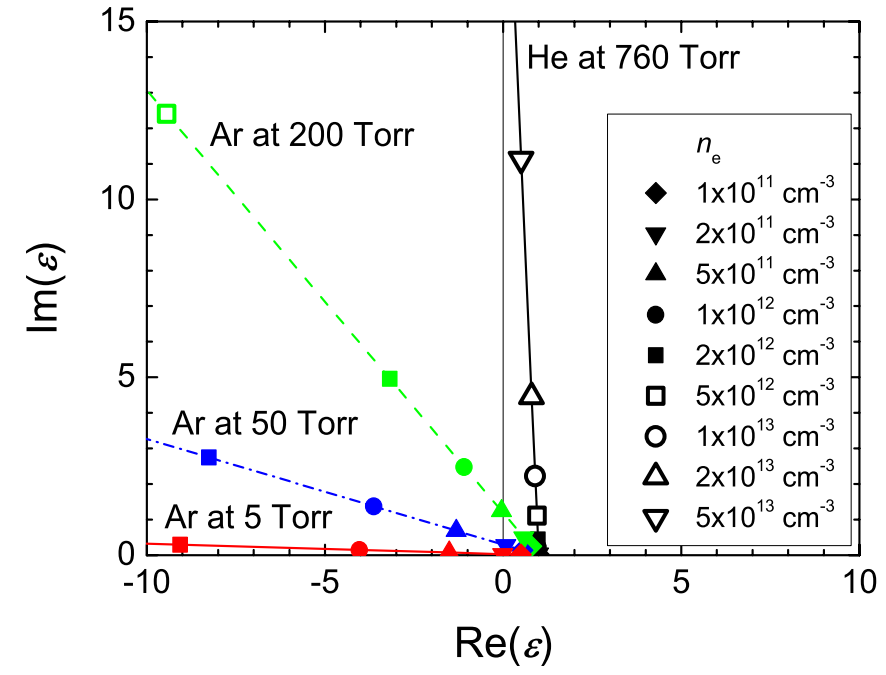

FIG. 1. (Color online) Permittivity in a lossy bulk plasma with various gas condition and various $n_{\mathrm{e}}$.

open new possibilities to control electromagnetic waves by complex-variable filters composed of microplasma assemblies.

Such physical aspects of plasma assemblies are reported in this article as follows. In Sec. II, we review dispersion relation in a plasma and its assembly, and a new drawing method of dispersion relation in 3D space of wave frequency, real and imaginary wavenumbers, is described. Here, we can explain fundamental properties on wave propagation, especially in a $2 \mathrm{D}$ periodic plasma structure. In Sec. III, experimental results are demonstrated in two different polarized waves. In Sec. IV, to reinforce theoretical prediction in Sec. II and to analyze experimental data in a more detailed manner, we show the finite difference time domain (FDTD) method applicable to plasma media and discuss the properties of the wave propagation in plasma assemblies, which is followed by a summary of this report in Sec. V.

\section{COMPLEX DISPERSION RELATIONS IN A PLASMA AND ITS ASSEMBLY}

To describe wave absorption as well as phase shift and/or reflection of the propagating waves, we here use a drawing of dispersion relation in the space in three coordinates consisting of wave frequency $\omega / 2 \pi$, real wavenumber $k_{\mathrm{r}}$, and imaginary wavenumber $k_{\mathrm{i}}$. A propagating wave which is launched at a spatial position $x=0$, or on the edge of a given media, is expressed as

$$
\begin{aligned}
A(x) \exp [j \phi(t, x)] & =A(x) \exp \left[j\left(\omega t-k_{\mathrm{r}} x\right)\right] \\
& =A_{0} \exp \left(k_{\mathrm{i}} x\right) \exp \left[j\left(\omega t-k_{\mathrm{r}} x\right)\right] \\
& =A_{0} \exp \left\{j\left[\omega t-\left(k_{\mathrm{r}}+j k_{\mathrm{i}}\right) x\right]\right\},
\end{aligned}
$$

where $A(x)$ is wave amplitude with the initial boundary condition of $A_{0}=A(x=0), t$ is time, and $\phi(t, x)$ is phase of the wave with the initial condition of $\phi(0,0)=0$. The dispersion relation in a collisionless plasma is usually expressed in the $\omega-k_{\mathrm{r}}$ plane, and we can also obtain a useful information
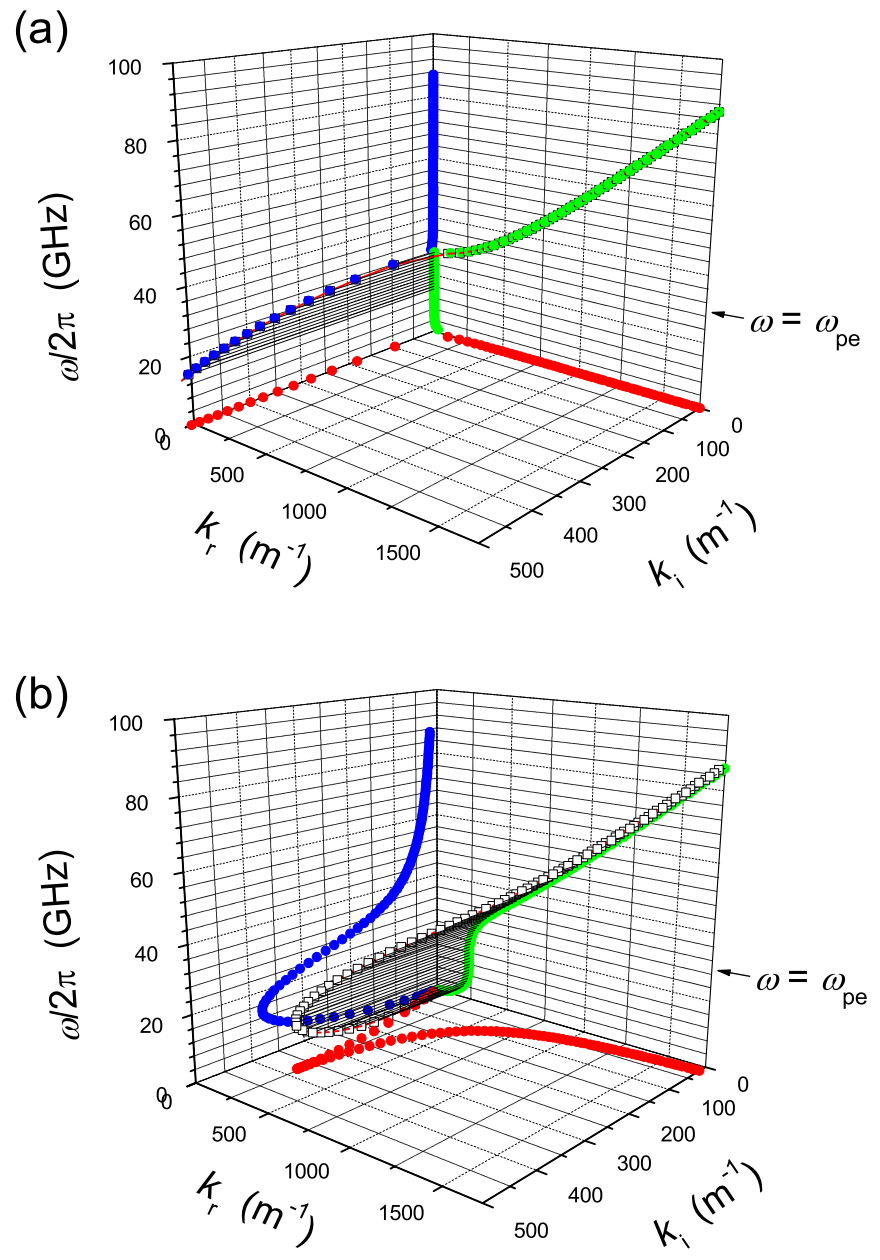

FIG. 2. (Color online) Dispersion relation of electromagnetic waves in a bulk plasma with $n_{\mathrm{e}}=1 \times 10^{13} \mathrm{~cm}^{-3}$ in the $3 \mathrm{D}$ space. (a) In a plasma at 5 Torr of Ar gas. (b) In a plasma at 120 Torr of He gas.

about wave attenuation from $k_{\mathrm{i}}$ as a function of $\omega$ when significant loss or wave attenuation takes place.

For instance, dispersion relation in a bulk nonmagnetized plasma is expressed by the permittivity $\varepsilon$ in the Drude model in the form

$$
\varepsilon=1-\frac{\omega_{\mathrm{pe}}^{2}}{\omega^{2}\left(1+j \nu_{\mathrm{m}} / \omega\right)},
$$

where $\omega_{\mathrm{pe}}$ is electron plasma frequency which is a function of electron density $n_{\mathrm{e}}$ and $\nu_{\mathrm{m}}$ is electron elastic collision frequency. Figure 1 shows $\varepsilon$ at a fixed wave frequency (4 GHz) as a function of $n_{\mathrm{e}}$ with various gas conditions on the complex plane. Here, we assume that electron energy is $0.5 \mathrm{eV}$ for a plasma in the afterglow and that cross section of electron elastic collisions is $5.0 \times 10^{-16} \mathrm{~cm}^{2}$ for $\mathrm{He}$ and $1.0 \times 10^{-16} \mathrm{~cm}^{2}$ for Ar from literature. ${ }^{24}$ At 760 Torr of He, $\operatorname{Re}(\varepsilon)$ is almost constant at unity for varying $n_{\mathrm{e}}$. On the other hand, at 5 Torr of $\operatorname{Ar}, \operatorname{Im}(\varepsilon)$ is almost zero while $\operatorname{Re}(\varepsilon)$ changes significantly in the negative polarity, and this feature almost corresponds to a collisionless plasma. This figure indicates that the change in gas species and pressure yields $\varepsilon$ with $\operatorname{Im}(\varepsilon) / \operatorname{Re}(\varepsilon)$ ranging from 0 to infinity for $\operatorname{Re}(\varepsilon)<1$ on the complex plane. 
Equation (2) gives us an understanding of dispersion relation in the 3D space $\left(\omega, k_{\mathrm{r}}, k_{\mathrm{i}}\right)$. Figure 2 displays dispersion relation in a bulk nonmagnetized plasma expressed by Eq. (2). In the case at 5 Torr of Ar, which is almost collisionless as mentioned earlier, the trajectory on the $\left(\omega, k_{\mathrm{r}}\right)$ plane is well known in literature. The working point is always on the $\left(\omega, k_{\mathrm{r}}\right)$ plane or on the $\left(\omega, k_{\mathrm{i}}\right)$ plane, which can be understood easily from Eq. (2). However, in the case at 120 Torr of He, the working point goes far away from the two planes below $\omega_{\mathrm{pe}}$ and leaves a trajectory on the $\left(k_{\mathrm{r}}, k_{\mathrm{i}}\right)$ plane; at such a point, the wave suffers attenuation as well as phase shift, as pointed out in Eq. (1).

Drawings of dispersion relation in this 3D space reveal significant physical parameters of electromagnetic media, as shown in the following. Knowledge from microwave engineering $^{25}$ shows that

$$
k=\omega \sqrt{\mu \varepsilon} \sqrt{1-j \frac{\sigma}{\omega \varepsilon}},
$$

where $\mu$ and $\sigma$ are permeability and conductivity of the media, respectively. From Eq. (3), the following equation is derived:

$$
k_{\mathrm{r}}\left|k_{\mathrm{i}}\right|=\frac{\omega \mu}{2} \sigma=\frac{1}{\delta_{\mathrm{s}}^{2}} .
$$

Here $\delta_{\mathrm{s}}$ is skin depth of the wave into the media. $k_{\mathrm{r}}\left|k_{\mathrm{i}}\right|$ indicates area on the $\left(k_{\mathrm{r}}, k_{\mathrm{i}}\right)$ plane, and so a point projected on the $\left(k_{\mathrm{r}}, k_{\mathrm{i}}\right)$ plane expresses conductivity of the media on the assumption that $\mu$ is constant. The inverse of the area on the $\left(k_{\mathrm{r}}, k_{\mathrm{i}}\right)$ plane corresponds to square of $\delta_{\mathrm{s}}$; as the area is larger, the skin depth is shorter. Another physical parameter that is visible in this 3D drawing is the metallic/dielectric boundary. From Eq. (3), we also obtain

$$
k_{\mathrm{r}}^{2}-k_{\mathrm{i}}^{2}=\omega^{2} \mu \varepsilon .
$$

Comprehension of this equation gives us the following result. If $k_{\mathrm{r}}>\left|k_{\mathrm{i}}\right|, \varepsilon$ is positive when $\mu$ is positive, leading to the fact that the media is dielectric, and if $k_{\mathrm{r}}<\left|k_{\mathrm{i}}\right|$, vice versa, and we can recognize that the media is metallic. The line of $k_{\mathrm{r}}=k_{\mathrm{i}}$ becomes the boundary between metallic and dielectric media.

These characteristics arising from lossy plasmas are distinguishable from other electromagnetic media; unlike plasmas, any other material never has a variety of parameter sets such as complex $\varepsilon$ and $\sigma$. Such a characteristic property can be enhanced by spatial periodicity; a simple periodic $\varepsilon_{\mathrm{r}}$ distribution realized in a solid material makes a photonic or electromagnetic band material, which includes photonic band gaps. If we introduce the effects of $\varepsilon_{\mathrm{i}}$ in a plasma array, new features can emerge with the complex-variable effects. A plasma array also works as an equivalent metal, which also affects photonic bands. We have already studied a part of its effects in our previous report, ${ }^{19}$ in which 1D structure was assumed. In the following, we investigated a 2D plasma assembly as an electromagnetic media with effects of a complex-variable filter. ${ }^{26}$
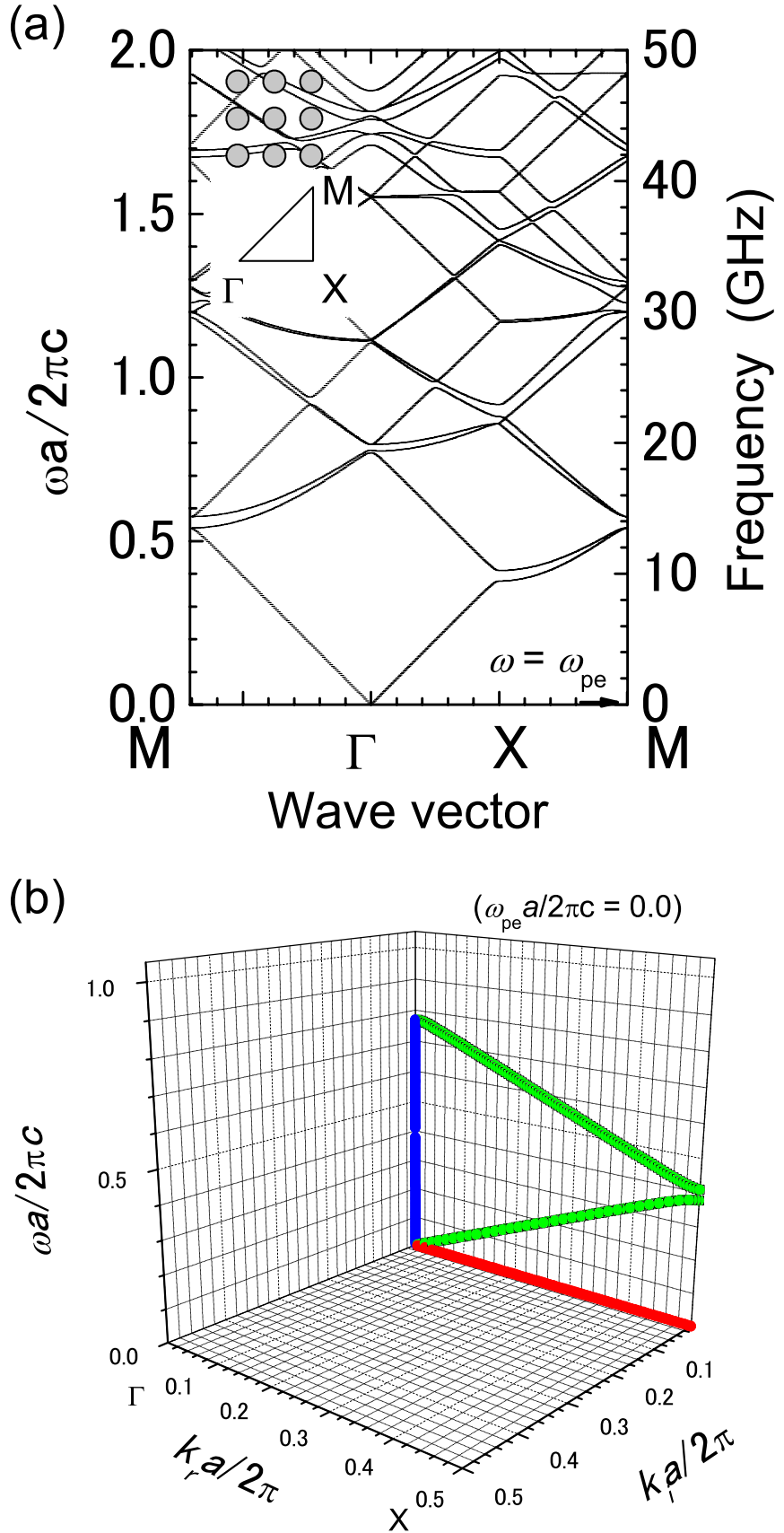

FIG. 3. (Color online) Dispersion relation derived using plane-wave expansion method in TE mode in a square lattice of $12 \mathrm{~mm}$ with filling factor of 0.18 . Vacuum holes are surrounded by dielectric with $\varepsilon=1.8$, and there is no plasma. (a) Photonic band of $k_{\mathrm{r}}$. (b) Branch around lowest photonic band gap in the $\Gamma-X$ direction in $3 \mathrm{D}$ space.

Before we consider a 2D plasma periodic structure, it would be useful to review photonic bands in a $2 \mathrm{D}$ dielectric periodic structure, where there is no frequency dependence in $\varepsilon$ that includes no imaginary part. Figure 3 shows a band diagram in a square lattice of vacuum holes formed in a bulk dielectric material with $\varepsilon=1.8$, calculated by the plane-wave expansion method. ${ }^{27,28}$ Although this method includes complicated formulas, the basic concept of the calculation is consistent with the Bloch ${ }^{29}$ or Froquet theorem, ${ }^{30}$ expressed in the forms of 


$$
\begin{aligned}
& \boldsymbol{E}\left(x_{0}+a\right)=\boldsymbol{E}\left(x_{0}\right) \exp \left(-j k_{x} a\right), \\
& \boldsymbol{E}\left(y_{0}+a\right)=\boldsymbol{E}\left(y_{0}\right) \exp \left(-j k_{y} a\right)
\end{aligned}
$$

and

$$
\begin{aligned}
& \frac{d \boldsymbol{E}}{d \boldsymbol{x}}\left(x_{0}+a\right)=\frac{d \boldsymbol{E}}{d \boldsymbol{x}}\left(x_{0}\right) \exp \left(-j k_{x} a\right), \\
& \frac{d \boldsymbol{E}}{d \boldsymbol{x}}\left(y_{0}+a\right)=\frac{d \boldsymbol{E}}{d \boldsymbol{x}}\left(y_{0}\right) \exp \left(-j k_{y} a\right),
\end{aligned}
$$

where $\boldsymbol{E}$ is an electric field of the propagating wave, $a$ is the lattice constant or the length of the spatial periodicity, and $\left(x_{0}, y_{0}\right)$ is a position vector $\boldsymbol{x}$ on the boundary of lattices. Here, coordinates $x$ and $y$ are along perpendicular sides of the lattice square. The calculated band diagram is an ordinary one of a photonic crystal, as shown in Fig. 3(a), and we recognize the first band gap along the $\Gamma-X$ direction in a frequency band, in which $a$ is around half wavelength in the dielectric. We note that Fig. 3(b) indicates no fraction of $k_{\mathrm{i}}$; in a 1D photonic crystal, there is a significant fraction of $k_{\mathrm{i}}$ in a dielectric photonic band gap, but in a 2D photonic crystal, the practical flow of wave energy diverges to the $\Gamma-M$ direction. $^{31}$

When we consider 2D structure composed of lossy microplasmas, the effects of dispersion in a plasma shown in Fig. 2 and of 2D spatial periodicity shown in Fig. 3 merged into distinctive photonic bands. A wave propagates in a $2 \mathrm{D}$ periodic structure in either mode: TE mode, in which wave electric fields are parallel to the 2D plane which is shown in the inset in Figs. 3(a), 4(a), and 5(a), and TM mode, in which wave magnetic fields are parallel to the $2 \mathrm{D}$ plane. The mode difference significantly affects photonic bands as shown below. Here we use the modified plane-wave expansion method $^{10}$ to derive photonic bands analytically. Figures 4 and 5 show photonic bands in both modes propagating in a columnar plasma array with a filling factor of 0.18 , periodic length of $12 \mathrm{~mm}$, slab density profile of $n_{\mathrm{e}}=1 \times 10^{13} \mathrm{~cm}^{-3}$, and $\nu_{\mathrm{m}}=0.5 \omega_{\mathrm{pe}}$. In the case of the TE mode, as shown in Fig. 4(a), a number of flatbands with very low group velocity are found below $\omega=\omega_{\text {pe }}$, arising from coupled surface-wave propagation. ${ }^{10,12}$ The lowest photonic band gap in the $\Gamma-X$ direction, which here we focus on, is located at 9-12.5 GHz. From Fig. 4(b), which is in the form of the 3D dispersion relation, this band gap also yields a kind of attenuation gaps, where $k_{\mathrm{i}}$ is discontinuous on the two sides of the frequency band gap. Additionally, smaller $k_{\mathrm{i}}$ is observed on the upper band; this feature is not consistent with the case in the 1D array. ${ }^{19}$ Such a behavior of the wave will be discussed using experimental results in Sec. III and numerical results in Sec. IV.

In the case of the TM mode, as shown in Fig. 5(a), no flatbands are observed, and the photonic bands around the lowest band gap in the $\Gamma-X$ direction are similar to those in a conventional dielectric photonic crystal. Figure 5(b) revealed discontinuity of $k_{\mathrm{i}}$ on the two sides of the band gap, although this attenuation gap is different from that in the TE mode; smaller $k_{\mathrm{i}}$ is observed on the lower band. This feature will (a)

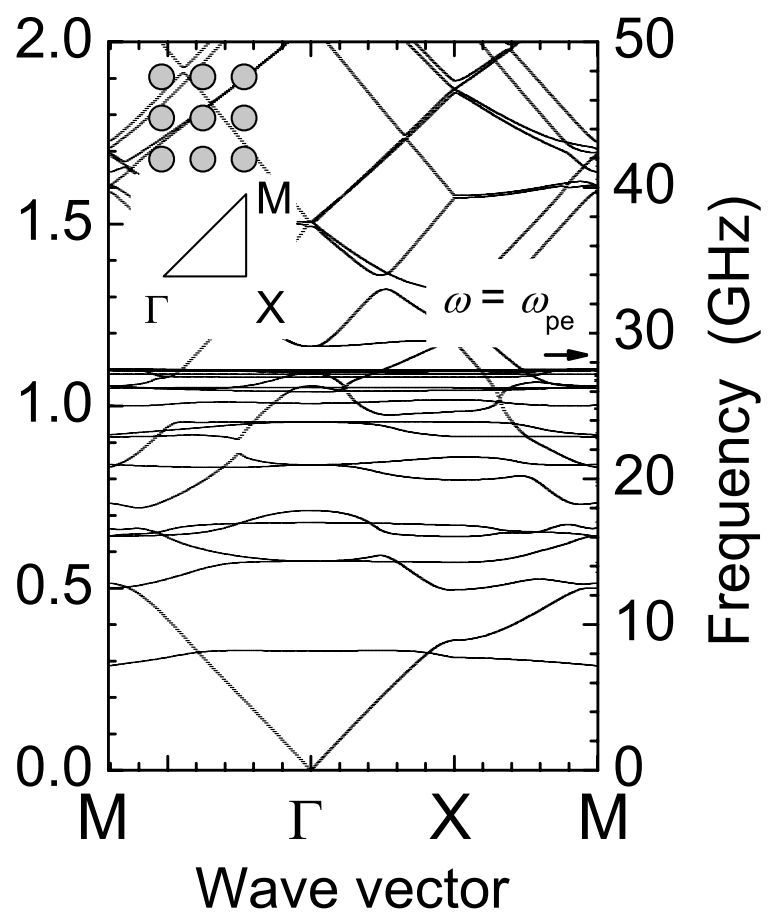

(b)

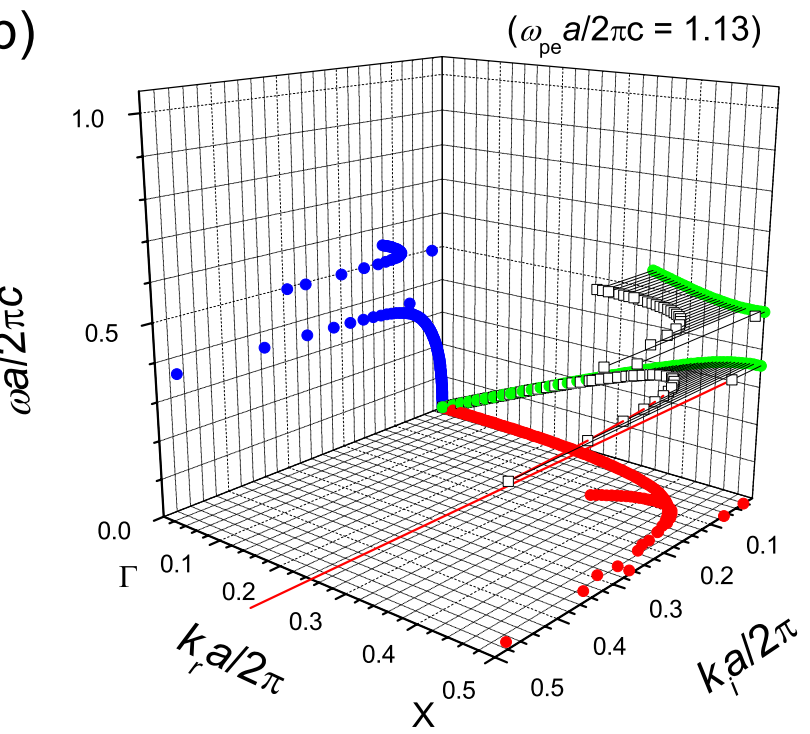

FIG. 4. (Color online) Dispersion relation derived using modified planewave expansion method in TE mode in a square lattice of $12 \mathrm{~mm}$ with filling factor of 0.18 and $n_{\mathrm{e}}=1 \times 10^{13} \mathrm{~cm}^{-3}$. (a) Photonic band of $k_{\mathrm{r}}$. (b) Branch around lowest photonic band gap except flatbands in the $\Gamma-X$ direction in $3 \mathrm{D}$ space.

also be discussed in Sec. IV. Another feature that was not observed in the TE mode is cutoff frequency around 8.0 $\mathrm{GHz}$. In a collisionless bulk plasma, there is a cutoff frequency at $\omega=\omega_{\text {pe }}$, as shown in Fig. 2(a). In the TM mode, this cutoff frequency is determined by a shield effect of the plasma columns whose axes are parallel to the electric field of the propagating waves; the cutoff mechanism is analogous to the Faraday shield used in a high-power wave launcher and to the long wavelength cutoff at a microwave waveguide. That is, this frequency is as a function of distance between plasma columns. On the other hand, in the TE 


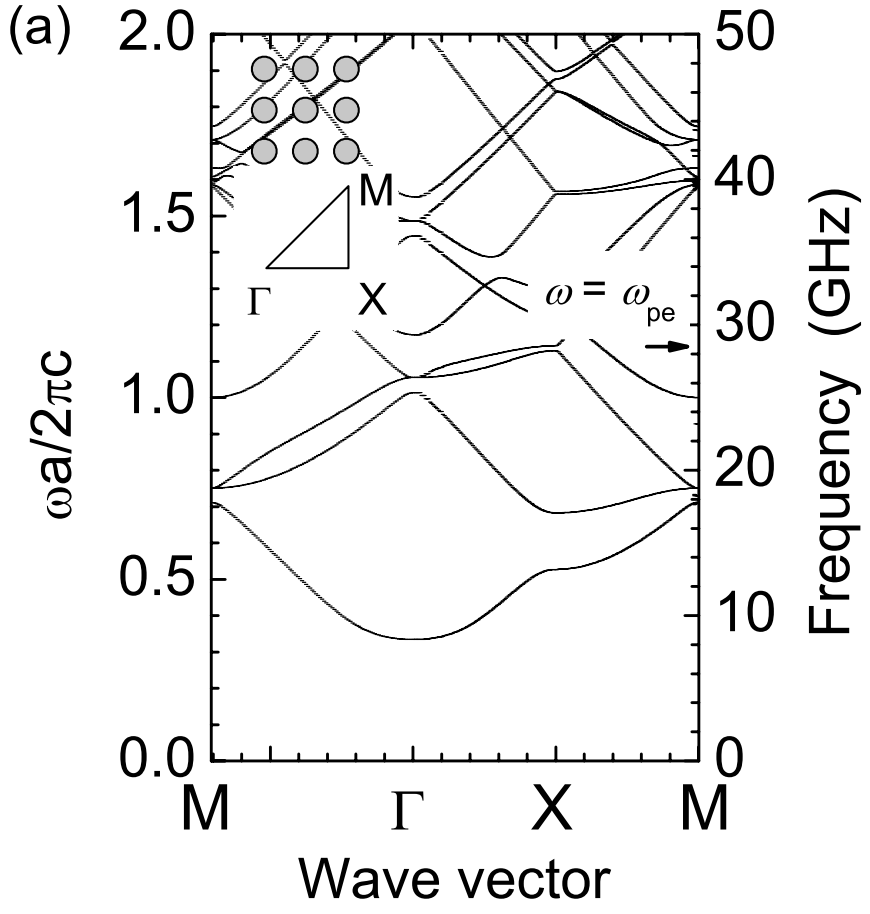

(b)

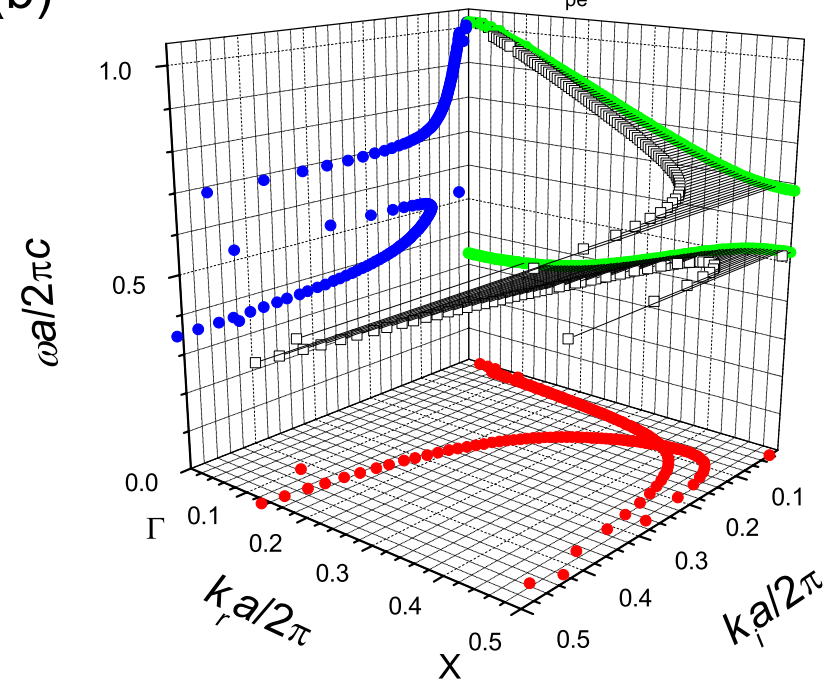

FIG. 5. (Color online) Dispersion relation derived using modified planewave expansion method in TM mode in a square lattice of $12 \mathrm{~mm}$ with filling factor of 0.126 and $n_{\mathrm{e}}=1 \times 10^{13} \mathrm{~cm}^{-3}$. (a) Photonic band of $k_{\mathrm{r}}$. (b) Branch around lowest photonic band gap in the $\Gamma-X$ direction in 3D space.

mode, electric fields can exist in a similar manner to the perpendicular fields to the metal components of a Faraday shield.

The common point in both modes is an effect of negative $\varepsilon$ on photonic bands modified by $\sigma$ or imaginary part of $\varepsilon$. This plasma assembly is equivalent to dielectric in the general frequency region even if the real part of $\varepsilon$ is negative, and it turns into a metallic media around the band gap in both modes and near the cutoff condition in the TM mode. In Secs. III and IV, we will figure out these analytical predictions in comparison with experimental and numerical results.

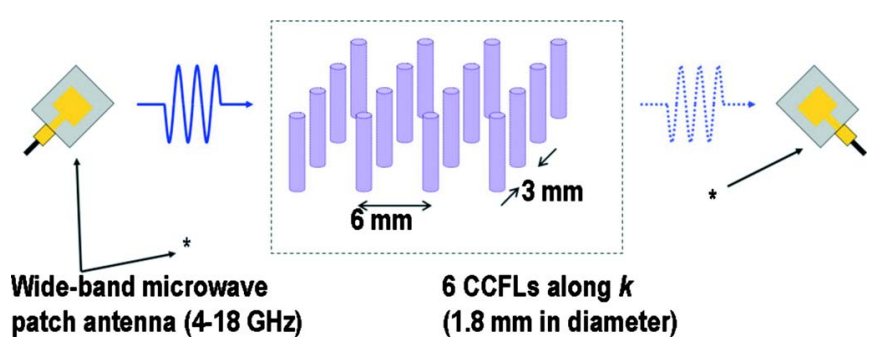

FIG. 6. (Color online) Experimental schematic setup for wave transmittance.

\section{EXPERIMENTAL METHOD}

\section{A. Experimental setup}

Since we investigate response of a plasma assembly in a wide range of microwaves from several gigahertz to around $20 \mathrm{GHz}$, a wideband antenna with good directionality is required for launching and propagation of quasiplane wave in a free space. Here we configured a new patch antenna based on a design reported recently, ${ }^{32}$ which enables us to obtain a fairly constant frequency spectrum from 4 to $18 \mathrm{GHz}$ with directionality of $\sim 20^{\circ}$ as a half of peak width in the main beam. Using this antenna, we measured amplitude of the microwaves propagating through a device under test consisting of capillaries for plasma columns, as shown in Fig. 6. From a signal generator (Agilent Inc., 83624B) through the patch antenna, microwaves were launched, and they were detected by a diode rectifier after received by the similar patch antenna, and the output signal of the detector was recorded in a digital storage oscilloscope.

The amplitude of the transmitted waves was normalized by that in the case without plasma generation, which was at $0 \mathrm{~dB}$ in the following experimental data. We refer to this value as transmittance in this report, and we pay attention to transmittance that is affected by both wave absorption and wave reflection; wave absorption arises from a lossy property of the plasma and reflection is a result of spatial discontinuity of real part of $\varepsilon$ and a band gap. To reveal each contribution to wave transmittance, we used phase measurement as well as detection of the amplitude of a transmitted wave in our previous report, ${ }^{19}$ although in this study we will use frequency dependence of the transmittance in comparison with numerical results.

A plasma column with diameter of $1.5 \mathrm{~mm}$ was generated in a CCFL capillary with outer diameter of $1.8 \mathrm{~mm}$, in which $\mathrm{Ar}$ and $\mathrm{Ne}$ gas mixture was contained with mercury and the working gas pressure was tens of Torrs, depending on vaporized mercury. Using similar CCFLs, we have successfully observed 1D dynamic photonic band gaps ${ }^{19}$ and surface-plasmon-like chains. ${ }^{20}$ To ignite plasmas in the capillaries, we applied bipolar square-shaped voltage pulses, where we fixed the monopolar pulse width to $7 \mu \mathrm{s}$, the repetition frequency to $20 \mathrm{kHz}$, and the discharge current in one capillary to $10 \mathrm{~mA}$ at maximum. In such an operational condition, electron density varied in time to a certain extent, although a plasma was continuously present. ${ }^{20}$ To obtain ex- 

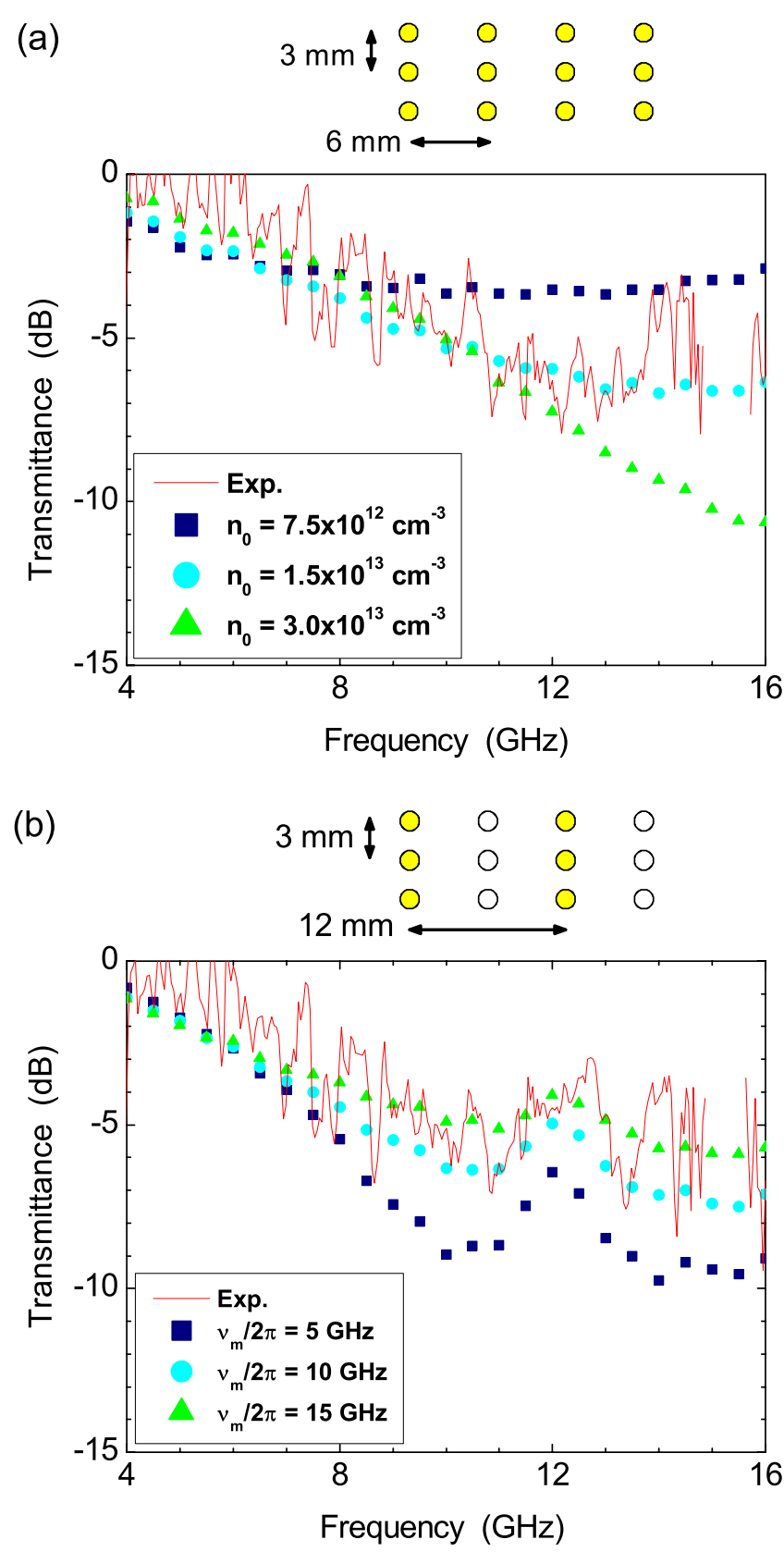

FIG. 7. (Color online) Experimental data of transmittance in TE mode (a) in $6 \mathrm{~mm}$ periodicity and (b) in $12 \mathrm{~mm}$. The dots indicates numerical results and we set $n_{\mathrm{e}}$ in the center of the column $=1.5 \times 10^{13} \mathrm{~cm}^{-3}$ and $\nu_{\mathrm{m}} / 2 \pi=12.5 \mathrm{GHz}$ if there are no specific notes.

perimental data shown in the following, we detected microwave signals at $30 \mu$ s after the rising slope of the repetitive positive monopolar pulse.

\section{B. Experimental results}

Figure 7(a) shows transmittance of the waves in the TE mode through the plasma assembly. Here, the assembly had a rectangular lattice of $3 \times 6 \mathrm{~mm}^{2}$, and along the wavenumber vector, in the $\Gamma$-X direction, spatial periodicity was $6 \mathrm{~mm}$. A photonic band gap was predicted to be above $20 \mathrm{GHz}$, and so the propagation in the lowest band was observed in this frequency range. The wave attenuation monotonously increased as the frequency was raised, which is a similar tendency to
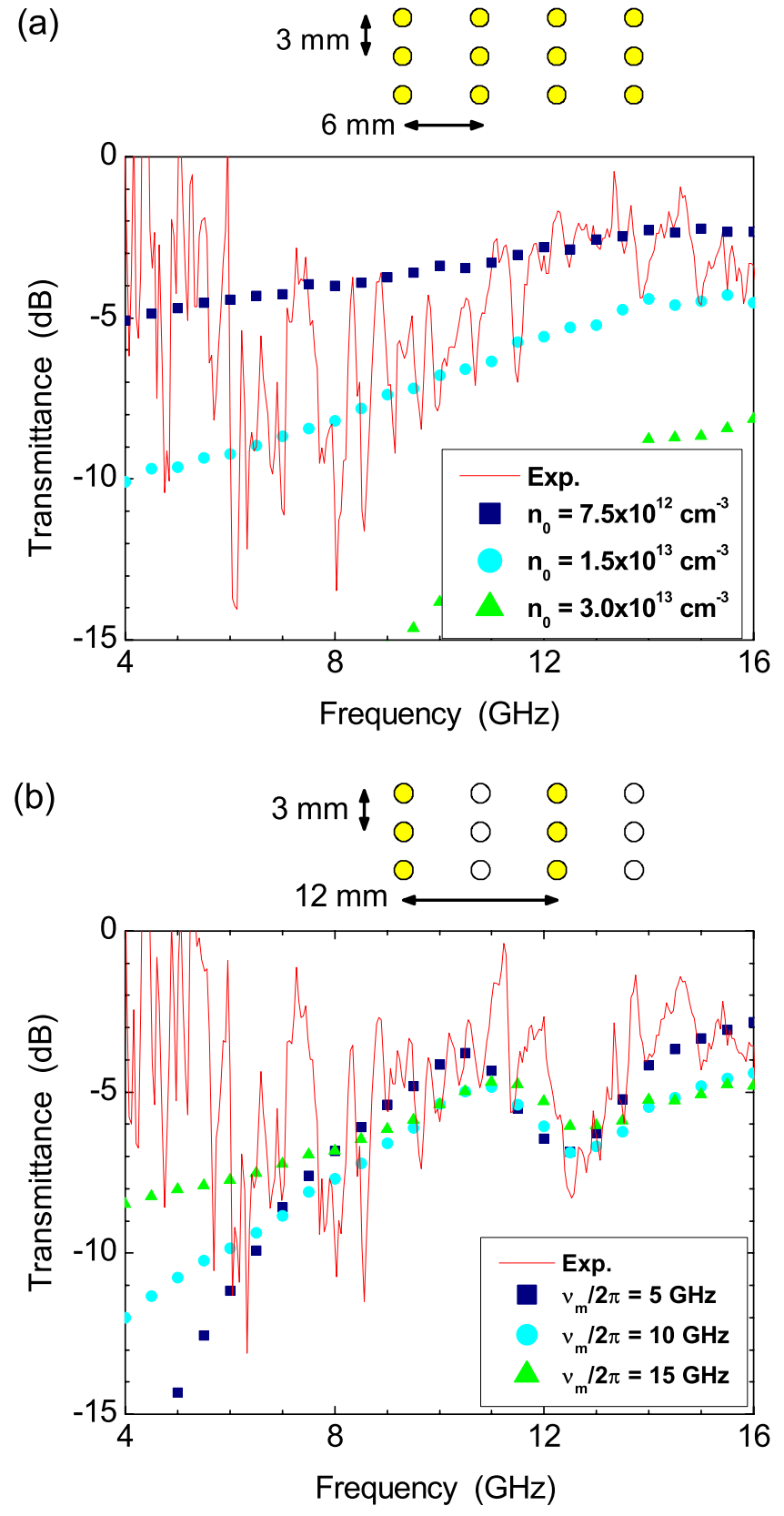

FIG. 8. (Color online) Experimental data of transmittance in TM mode (a) in $6 \mathrm{~mm}$ periodicity and (b) in $12 \mathrm{~mm}$. The dots indicates numerical results and we set $n_{\mathrm{e}}$ in the center of the column $=1.5 \times 10^{13} \mathrm{~cm}^{-3}$ and $\nu_{\mathrm{m}} / 2 \pi=12.5 \mathrm{GHz}$ if there are no specific notes.

the predicted $k_{\mathrm{i}}$ below $\omega a / 2 \pi c \sim 0.3$ in Fig. 4(b). Figure 7(b) shows transmittance in the case of a rectangular capillary lattice of $3 \times 6 \mathrm{~mm}^{2}$ with the spatial periodicity of $12 \mathrm{~mm}$ along the wavenumber vector; that is, plasmas were generated in every two rows. This case is roughly similar to the wave propagation around the lowest band gap, i.e., for $0.2<\omega a / 2 \pi c<0.6$, examined in Fig. 4(b). Figure 4(b) shows discontinuity of $k_{\mathrm{i}}$ around the band gap, and this phenomenon was also observed for $11-13 \mathrm{GHz}$ as a reduction in wave attenuation in Fig. 7(b).

Figure 8(a) shows transmittance of the waves in the TM mode through the plasma assembly. The pattern of plasma ignition in CCFLs was similar to that in Fig. 7; the assembly 
had a rectangular lattice of $3 \times 6 \mathrm{~mm}^{2}$, and along the wavenumber vector the spatial periodicity was $6 \mathrm{~mm}$. Since the photonic band gap was predicted to be above $20 \mathrm{GHz}$, the propagation in the lowest band was observed in this frequency range. In comparison with the cases in the TE mode, we observed a couple of different points; the wave attenuation decreased as the frequency was raised, which is a similar tendency to the predicted $k_{\mathrm{i}}$ below $\omega a / 2 \pi c \sim 0.3$ in Fig. 5(b). Figure 8(b) shows transmittance in the case of a rectangular capillary lattice of $3 \times 6 \mathrm{~mm}^{2}$ with the spatial periodicity of $12 \mathrm{~mm}$ along the wavenumber vector, where the plasmas were generated in every two rows. This case is also roughly similar to the wave propagation around the lowest band gap, i.e., for $0.2<\omega a / 2 \pi c<0.6$, examined in Fig. 5(b). Figure 5(b) shows discontinuity of $k_{\mathrm{i}}$ around the band gap, and this phenomenon was also observed for 9-13 GHz in Fig. 8(b), where both enhancement as well as reduction in wave attenuation were observed. This enhancement was supposed to arise from the photonic frequency band gap itself. In the following, in Sec. IV, we comprehend these experimental and theoretical results described in Secs. II and III using numerical results.

In these experimental results one may recognize that the signal-to-noise ratio is not so large at some frequencies. This is partially attributed to standing waves between the antenna and/or glass capillaries with microplasmas. Another possibility is anisotropic directionality of our newly developed wideband antenna; the directionality depends on wave frequency and so at some frequencies, excessive reflected waves from outside objects may come into the receiver antenna. However, at general frequencies, the detected signals are fairly consistent with numerical results, which will be described in Sec. IV in detail.

\section{NUMERICAL ANALYSIS}

\section{A. Numerical method}

As a numerical method for analysis of electromagnetic waves propagating in a plasma assembly, we developed a FDTD method ${ }^{33}$ applicable to plasma media. A FDTD method has been widely used for analysis of waves propagating in 2D or 3D structure, and we introduce a couple of points to a conventional FDTD method to treat plasma effects in a rigorous manner.

Maxwell equations are linearized according to Yee's algorism, ${ }^{34}$ as used in a conventional FDTD method. In addition, the following equation is combined with Maxwell equations in the similar dicretization manner: ${ }^{35}$

$$
\frac{\partial \boldsymbol{J}}{\partial t}+\nu_{\mathrm{m}} \boldsymbol{J}=\varepsilon_{0} \omega_{\mathrm{pe}}{ }^{2}(\boldsymbol{r}) \boldsymbol{E},
$$

where $\boldsymbol{J}$ and $\boldsymbol{E}$ are a current density and an electric field of the wave, respectively, and $\boldsymbol{r}$ is the position vector. Here, we ignored a pressure-gradient term from the general momentum balance equation in the fluid model because the pressure-gradient term is in the order of $10^{-7}$ of the righthand side of Eq. (8), although we have to treat it rigorously when electron temperature is quite high or when electromagnetic waves propagate with short wavelength such as surface

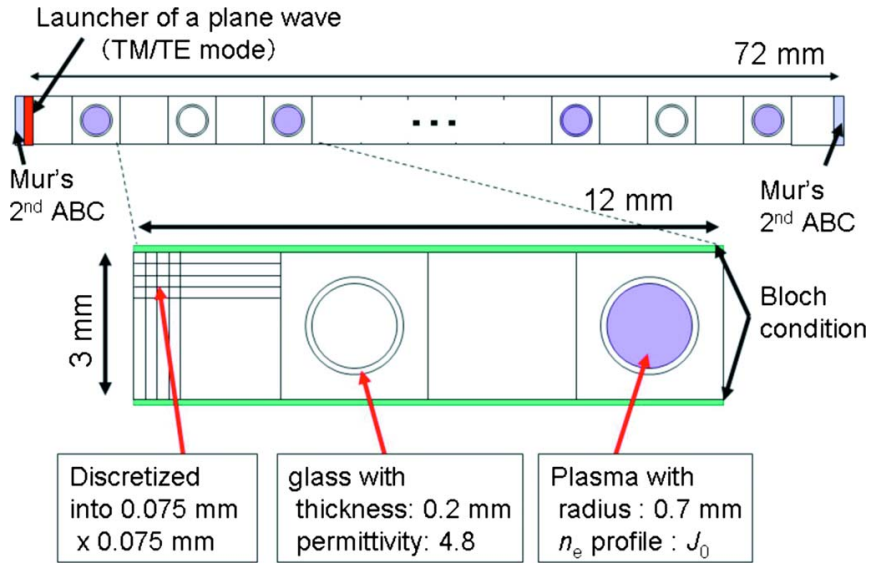

FIG. 9. (Color online) Schematic of numerical modeling space of FDTD method.

waves. The addition of Eq. (8) to the conventional FDTD method allows us to deal a plasma with an arbitrary $n_{\mathrm{e}}$ profile, and we adapted a Bessel function of the first kind in the zeroth order $J_{0}$ in the following since our plasmas in the experiments were in the regime of the positive column with $n_{\mathrm{e}}=0$ on the edge. ${ }^{36} \mathrm{We}$ also ignored a diffusion term from the general fluid model because particle displacement due to the diffusion flux in our plasma parameters within the calculation time was quite short in comparison with the plasma size assumed in the calculation.

Figure 9 shows a configuration of the numerical modeling. Waves were launched near the left end of the model domain and propagated toward the right direction. The boundary layers behind the wave launcher and on the right end were in Mur's second absorption boundary condition. ${ }^{37}$ The upper and lower boundary layers fulfill the $\mathrm{Bloch}^{29}$ or Froquet theorem ${ }^{30}$ to assure the spatial periodic structure.

By the modified plane-wave expansion method described in Sec. II, we derived dispersion relation or photonic bands in an infinite square lattice of plasmas with the slab- $n_{\mathrm{e}}$ profile. Using this FDTD method, specific calculation in the similar condition to experiments was available; we could calculate wave propagation in a finite-size array including a rectangular lattice, consisting of plasmas with an arbitrary density profile. Furthermore, as shown in Figs. 10 and 11, profiles of each field were simultaneously obtained, which may yield comprehension of detailed propagation mechanisms.

\section{B. Numerical results and discussion}

Numerical data on transmittance of the calculated wave propagation were shown in Figs. 7 and 8 as dot symbols. Here we set parameters around $n_{\mathrm{e}}$ in the center of the column $=1.5 \times 10^{13} \mathrm{~cm}^{-3}$ and $\nu_{\mathrm{m}} / 2 \pi=12.5 \mathrm{GHz}$, as shown in Figs. 7 and 8. The numerical data matched the experimental data very well in any case if we set $n_{\mathrm{e}}$ in the center of the column $=1.5 \times 10^{13} \mathrm{~cm}^{-3}$ and $\nu_{\mathrm{m}} / 2 \pi=12.5 \mathrm{GHz}$, which are the estimated plasma parameters deduced from this comparison.

This good consistency enables us to analyze propagating waves around the lowest band gap, more specifically when 


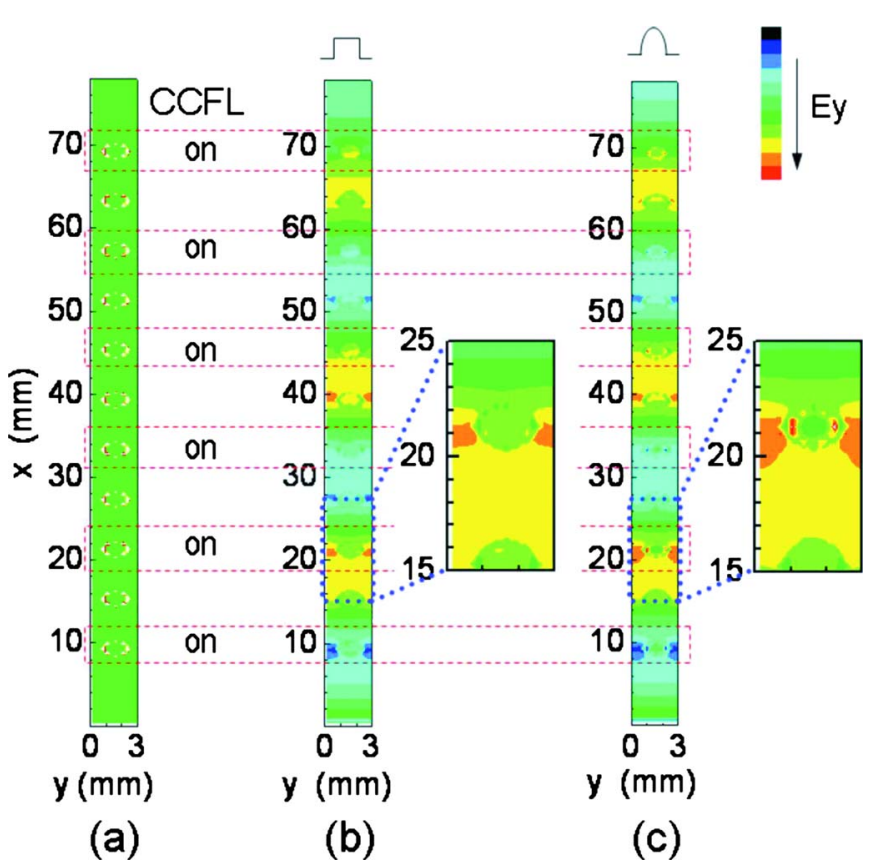

FIG. 10. (Color online) Electric field profiles in TE mode numerically derived in condition similar to Fig. 5. (a) CCFL position, where plasma is generated in every two CCFL with periodic length of $12 \mathrm{~mm}$, (b) electric field parallel to 2D plane in the case with slab $n_{\mathrm{e}}$ profile, and (c) electric field parallel to 2D plane in the case with $n_{\mathrm{e}}$ profile with $J_{0}$.

we take a close look at numerically obtained field profiles. Figures 10 and 11 display profiles of electric field amplitude of the TE and TM modes when the wave attenuation was reduced significantly. We showed the field profiles in the case of the slab $n_{\mathrm{e}}$ profile in comparison. In both modes, since the frequencies were in the vicinity of the lowest band gap in which the spatial periodic length is the half of the wavelength, the wave amplitude changed in a synchronous variation with the plasma patterns; the position of the maximum amplitude overlapped the distributed $n_{\mathrm{e}}$ area. In the case of the TE mode at $12.5 \mathrm{GHz}$, where the wave attenuation was reduced significantly, the electric field got away from the high $n_{\mathrm{e}}$ region in the center of the plasmas, which leads to the reduction in the wave attenuation observed in the experiment. This feature also coincides with $k_{\mathrm{i}}$ on the upper side of the band gap in Fig. 4. We also pointed out that in the TE mode, a profile of electric field may be heavily affected by the $n_{\mathrm{e}}$ profile; in the case of $J_{0}$ profile, waves sneaked into the plasma region where they might suffer a certain damping.

In the case of the TM mode at $11.0 \mathrm{GHz}$, where the wave attenuation was reduced significantly, the electric field that was along the plasma column axis was distributed without the effects of the $n_{\mathrm{e}}$ region, as shown in Fig. 11. In this case, the magnetic field is parallel to the plane of the cross section and was not directly affected by the spatial change of $\varepsilon$. This difference in the wave propagation gives rise to the change in wave propagation between these modes.

When we review Figs. 7 and 8, one significant difference is the clearer enhancement of wave attenuation in the band gap in the TM mode. One possible explanation for this phenomenon is the presence of waves on the flatbands in the TE

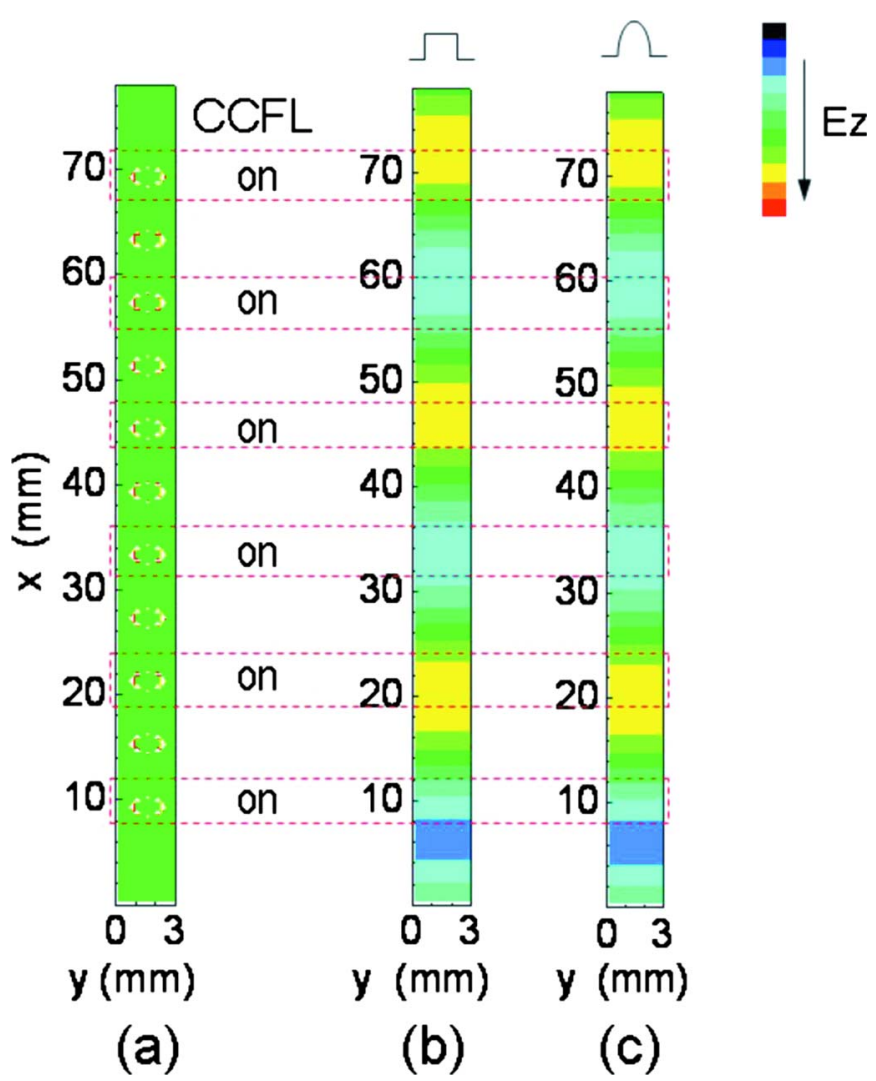

FIG. 11. (Color online) Electric field profiles in TM mode numerically derived in condition similar to Fig. 6. (a) CCFL position, where plasma is generated in every two CCFL with periodic length of $12 \mathrm{~mm}$, (b) electric field parallel to 2D plane in the case with slab $n_{\mathrm{e}}$ profile, and (c) electric field parallel to 2D plane in the case with $n_{\mathrm{e}}$ profile with $J_{0}$.

mode; Fig. 4 indicates that one or a few flatbands might be present around or in the lowest band gap and they might contribute to wave propagation.

One may think that replacement of microplasmas with metals of the similar size will clarify effects of plasmas on photonic bands in a simpler way; however, it is usually very difficult to analyze such experimental results. Several previous studies told us that a 2D metallic array works as an artificial dielectric $^{38}$ and/or a metamaterial ${ }^{39}$ at microwaves. Experimental data include these two effects at least and more complicated inductance-capacitance resonance phenomena in some cases. Our current conclusion about methods for comprehension of dispersion relation of waves propagating a microplasma array is that the numerical and analytical approaches described in this manuscript are fairly appropriate.

These results of the two polarization modes suggest that selection of polarization is another tool to control wave amplitude and phase shift independently. Up until now, we can use three different schemes to control such wave parameters by plasma assemblies: a set of $n_{\mathrm{e}}$ and gas pressure, spatial periodicity, and wave polarization. Recently, we realized a complex-variable filter with a simple function such as "sum" using 1D plasma assemblies by changing their spatial periodicity. ${ }^{26}$ By selecting an adequate tool for an individual application device, we can control electromagnetic-wave 
propagation on the complex plane, which will lead to a compact wave controller and/or detector at frequencies ranging from microwaves to terahertz waves.

\section{CONCLUSION}

In this report, we clarified wave propagation in a plasma assembly whose permittivity was expressed in a complex variable. After pointing out the significance of the dispersion relation drawn in the $3 \mathrm{D}$ space, fundamental properties that assured importance of plasmas as electromagnetic media were demonstrated. The band diagrams derived by the modified plane-wave expansion method predicted wave propagation in a plasma assembly, and they were verified in the experimental results. The numerical calculation based on the conventional FDTD method and modified to fulfill requirements for plasmas enabled us to recognize consistency with the experimental results. The potential of the plasmas as novel electromagnetic media and these methods developed so far to clarify their properties will lead to the future development for arbitrary complex-variable filters for microwaves and possibly terahertz waves.

\section{ACKNOWLEDGMENTS}

This work was supported in part by the Industrial Technology Research Grant Program of 2006 from the New Energy and Industrial Technology Development Organization (NEDO) of Japan.

${ }^{1}$ K. Tachibana, IEEJ Trans. Electr. Electron. Eng. 1, 145 (2006).

${ }^{2}$ F. Iza, G. J. Kim, S. M. Lee, J. K. Lee, J. L. Walsh, Y. T. Zhang, and M. G. Kong, Plasma Processes Polym. 5, 322 (2008).

${ }^{3}$ T. H. Stix, The Theory of Plasma Waves (McGraw-Hill, New York, 1962).

${ }^{4}$ V. L. Ginzburg, The Propagation of Electromagnetic Waves in Plasma (Pergamon, Oxford, 1964).

${ }^{5}$ D. G. Swanson, Plasma Waves (Academic, Boston, 1989).

${ }^{6}$ J. Faith, S. P. Kuo, and J. Huang, Phys. Rev. E 55, 1843 (1997).

${ }^{7}$ G. G. Borg, J. H. Harris, N. M. Martin, D. Thorncraft, R. Milliken, D. G. Miljak, B. Kwan, T. Ng, and J. Kircher, Phys. Plasmas 7, 2198 (2000).

${ }^{8}$ H. Hojo and A. Mase, J. Plasma Fusion Res. 80, 89 (2004).
${ }^{9}$ O. Sakai, T. Sakaguchi, and K. Tachibana, Appl. Phys. Lett. 87, 241505 (2005).

${ }^{10}$ O. Sakai, T. Sakaguchi, and K. Tachibana, J. Appl. Phys. 101, 073304 (2007).

${ }^{11}$ T. Sakaguchi, O. Sakai, and K. Tachibana, J. Appl. Phys. 101, 073305 (2007).

${ }^{12}$ O. Sakai and K. Tachibana, IEEE Trans. Plasma Sci. 35, 1267 (2007).

${ }^{13}$ D. K. Kalluri, Electromagnetics of Complex Media (CRC, Boca Raton, 1998).

${ }^{14}$ V. Kuzmiak and A. A. Maradudin, Phys. Rev. B 55, 7427 (1997).

${ }^{15}$ O. Sakai, Y. Kishimoto, and K. Tachibana, J. Phys. D 38, 431 (2005).

${ }^{16}$ O. Sakai, T. Sakaguchi, Y. Ito, and K. Tachibana, Plasma Phys. Controlled Fusion 47, B617 (2005).

${ }^{17}$ E. E. Kunhardt, IEEE Trans. Plasma Sci. 28, 189 (2000).

${ }^{18}$ O. Sakai, T. Sakaguchi, T. Naito, D.-S. Lee, and K. Tachibana, Plasma Phys. Controlled Fusion 49, B453 (2007).

${ }^{19}$ T. Naito, O. Sakai, and K. Tachibana, Appl. Phys. Express 1, 066003 (2008).

${ }^{20}$ O. Sakai, T. Naito and K. Tachibana, J. Plasma Fusion Res. 4, 052 (2009).

${ }^{21}$ T. Shirafuji, T. Kitagawa, T. Wakai, and K. Tachibana, Appl. Phys. Lett. 83, 2309 (2003).

${ }^{22}$ S. N. Abolmasov, T. Shirafuji, and K. Tachibana, IEEE Trans. Plasma Sci. 33, 941 (2005).

${ }^{23}$ L. F. Dong, Y. F. He, W. L. Liu, R. L. Gao, H. F. Wang, and H. T. Zhao, Appl. Phys. Lett. 90, 031504 (2007).

${ }^{24}$ S. C. Brown, Basic Data of Plasma Physics (MIT Press, Boston, 1959).

${ }^{25}$ D. M. Pozar, Microwave Engineering (Addison-Wesley, Reading, 1990).

${ }^{26}$ O. Sakai, T. Naito, T. Shimomura, and K. Tachibana, "Microplasma array with metamaterial effects," Thin Solid Films (to be published).

${ }^{27}$ K. M. Ho, C. T. Chan, and C. M. Soukoulis, Phys. Rev. Lett. 65, 3152 (1990).

${ }^{28}$ M. Plihal, A. Shambrook, A. A. Maradudin, and P. Sheng, Opt. Commun. 80, 199 (1991).

${ }^{29}$ F. Bloch, Z. Phys. A 52, 555 (1929).

${ }^{30}$ G. Floquet, Ann. Sci. Ec. Normale Super. 12, 47 (1883).

${ }^{31}$ O. Sakai, T. Sakaguchi, and K. Tachibana, Contrib. Plasma Phys. 47, 96 (2007).

${ }^{32}$ S. H. Choi, J. K. Park, S. K. Kim, and J. Y. Park, Microwave Opt. Technol. Lett. 40, 399 (2004).

${ }^{33}$ K. S. Kunz and R. J. Luebbers, The Finite Difference Time Domain Method for Electromagnetics (CRC, Boca Raton, 1993); J. H. Lee and D. K. Kalluri, IEEE Trans. Antennas Propag. 47, 1146 (1999).

${ }^{34}$ K. Yee, IEEE Trans. Antennas Propag. 14, 302 (1966).

${ }^{35}$ J. L. Young, Radio Sci. 25, 1913 (1994).

${ }^{36}$ A. von Engel, Ionized Gases (Clarendon, Oxford, 1965).

${ }^{37}$ G. Mur, IEEE Trans. Electromagn. Compat. EMC-23, 377 (1981).

${ }^{38}$ W. Rotman, IRE Trans. Antennas Propag. 10, 82 (1962).

${ }^{39}$ J. B. Pendry, A. J. Holden, W. J. Stewart, and I. Yougns, Phys. Rev. Lett. 76, 4773 (1996). 